\title{
Ductilization of Nanoporous Ceramics by
}

\section{Crystallinity Control}

\section{- Supporting Information -}

Jung Woo Kim ${ }^{1}$, Dahye Shin ${ }^{1}$, Yang Jeong Park ${ }^{1}$, Dongchan Jang ${ }^{1^{*}}$, and Sung Oh Cho ${ }^{{ }^{*}}$

${ }^{1}$ Department of Nuclear and Quantum Engineering, Korea Advanced Institute of Science and Technology, Daejeon, 34141, Republic of Korea

*e-mail: dongchan.jang@kaist.ac.kr; socho@kaist.ac.kr

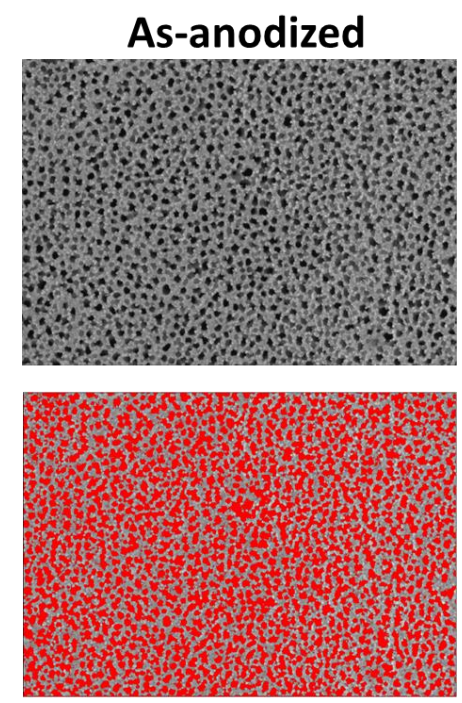

$45.83 \%$
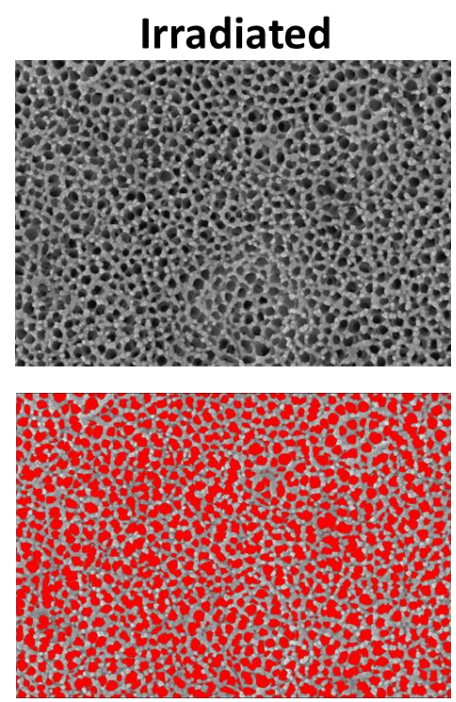

$44.52 \%$
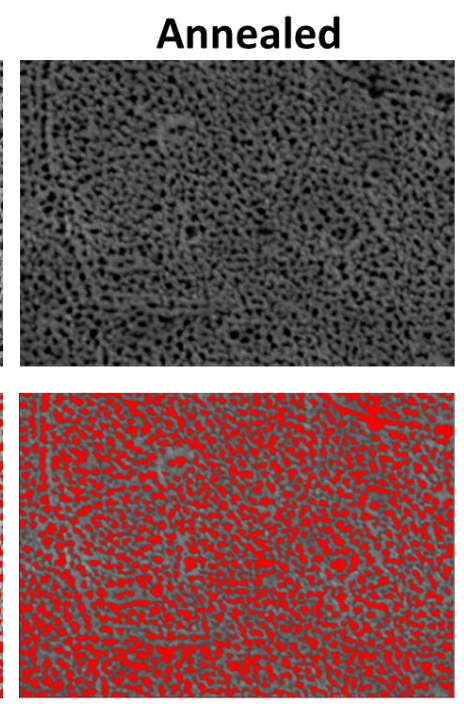

$34.09 \%$

Figure S1. Calculated values of porosity of each condition utilizing image $\mathrm{j}$ software.

The values of porosity for as-anodized, irradiated and annealed oxide films were calculated using image $\mathrm{j}$ software as shown in Figure S1. Both as-anodized and irradiated oxide films 
were estimated to be about $45 \%$ porosity. Meanwhile, this porosity value was decreased by about $10 \%$ after annealing process.
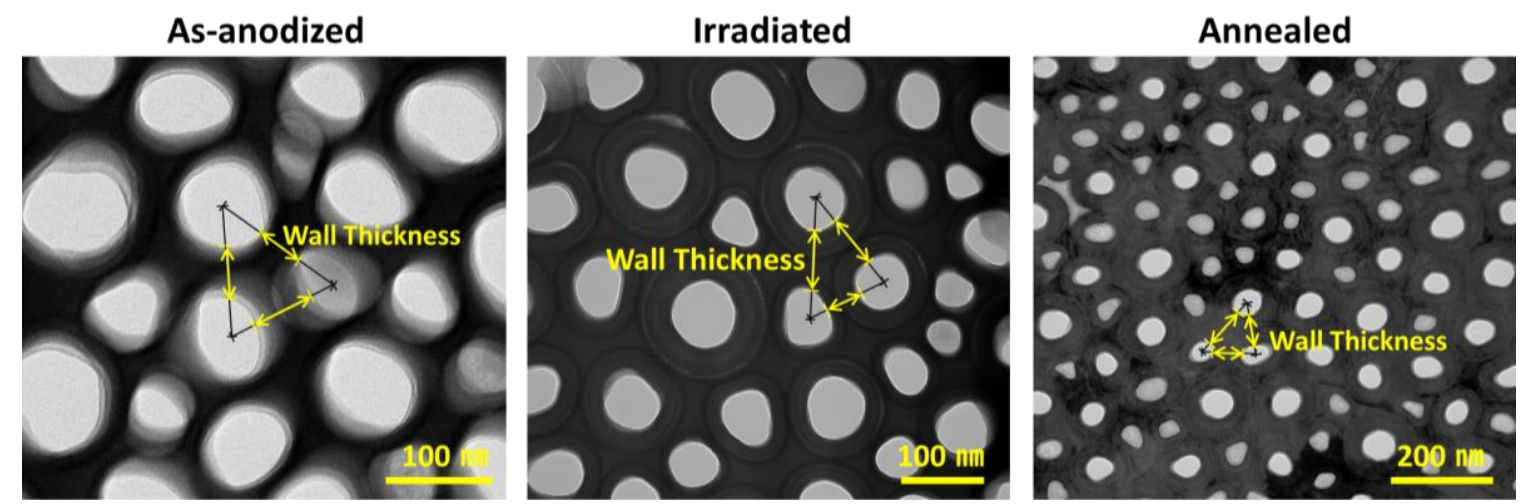

Figure S2. Low magnification TEM images of as-anodized, irradiated and annealed nanoporous oxide films.

\begin{tabular}{cc}
\hline Wall thickness & $\begin{array}{c}\text { Average } \\
(\mathrm{nm})\end{array}$ \\
\hline As-anodized & $47.62 \pm 11.38$ \\
\hline Irradiated & $47.34 \pm 13.58$ \\
\hline Annealed & $56.92 \pm 11.95$ \\
\hline
\end{tabular}

Table S1. Wall thickness of as-anodized, irradiated and annealed nanoporous oxide fi $\operatorname{lms}$.

Low magnification TEM images of each film were presented in Figure S2 for estimating the wall thickness. The wall thickness was estimated as a distance occupied by the solid along the line connecting two neighboring pore centers. The average values of wall thickness of asanodized, irradiated and annealed porous structures were summarized in Table S1. These average values were obtained by sampling 60, 92 and 151 different thicknesses in as-anodized, irradiated, and annealed oxide films, respectively.

(a)

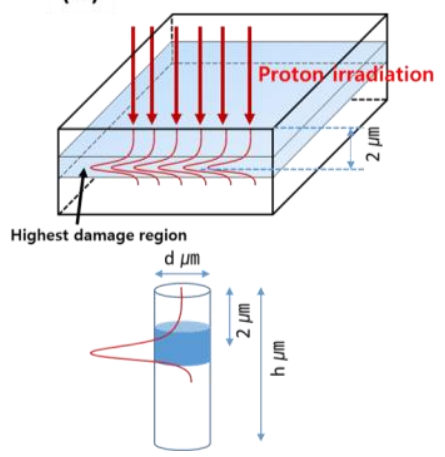

(b)

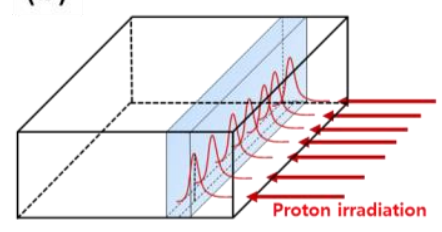

$\stackrel{\mathrm{d} \mu \mathrm{m}}{\longrightarrow}$

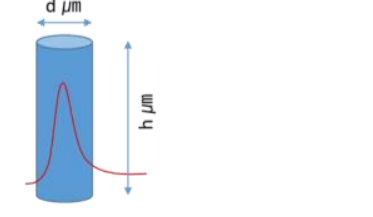

(c)

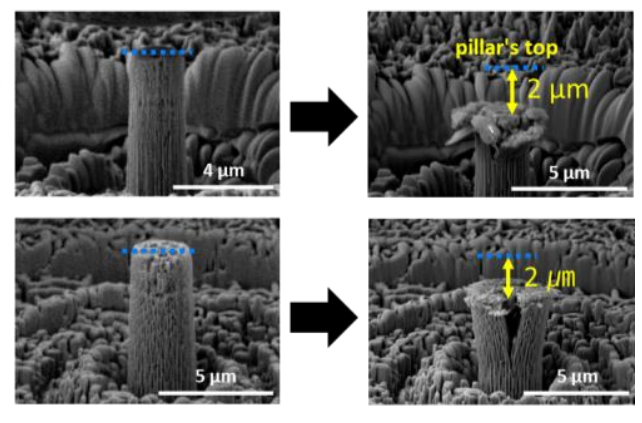

Figure S3. Schematic of perpendicular (a), horizontal (b) irradiation and SEM images of fractured micro-pillars (c) which perpendicularly irradiated. 
In Figure S3, the schematic shows that the highest damage region depends on the direction of proton irradiation. When vertically irradiated, a considerable amount of ion displacements was concentrated at a position near $2 \mu \mathrm{m}$ from the pillar's top. Since this region became locally fragile, the mechanical response with irradiation effects could not be obtained due to the failure at that region before all mechanical responses were yielded during the compression. As a result, it was observed that breakage occurred at the similar regions of the compressed micro-pillars in common (Figure S3c). On the other hand, when horizontally irradiated (perpendicular to the pore channel axis), micro-pillars without radiation damage gradient can be obtained (Figure $\mathrm{S} 3 \mathrm{~b})$.

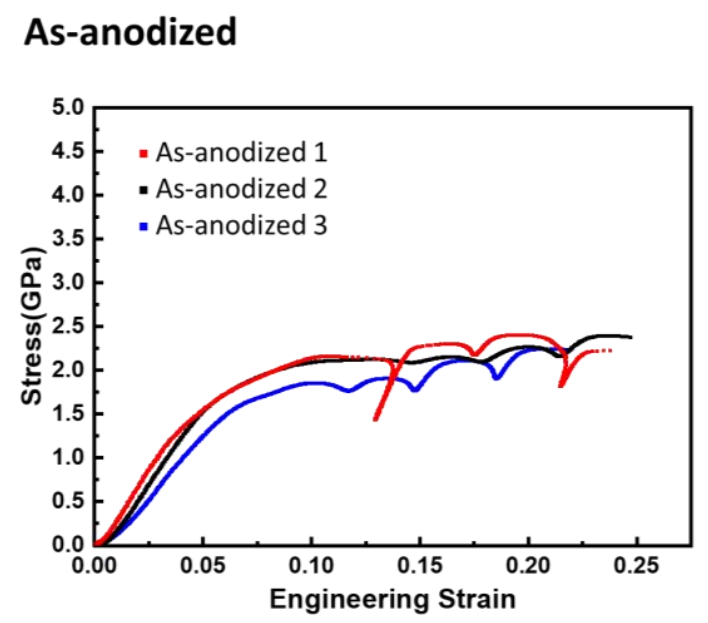

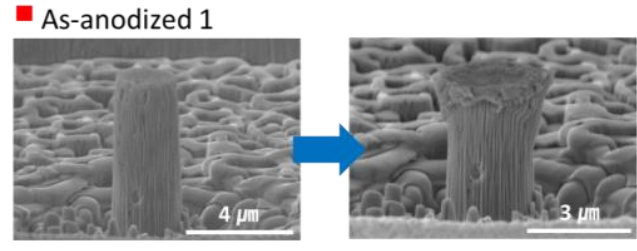
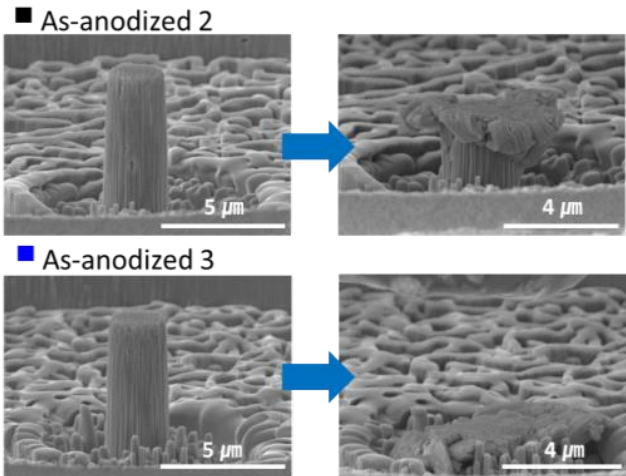

Figure S4. Engineering stress-strain curves of micro-compression test and SEM imag es of before and after compressed micro-pillar for as-anodized condition.
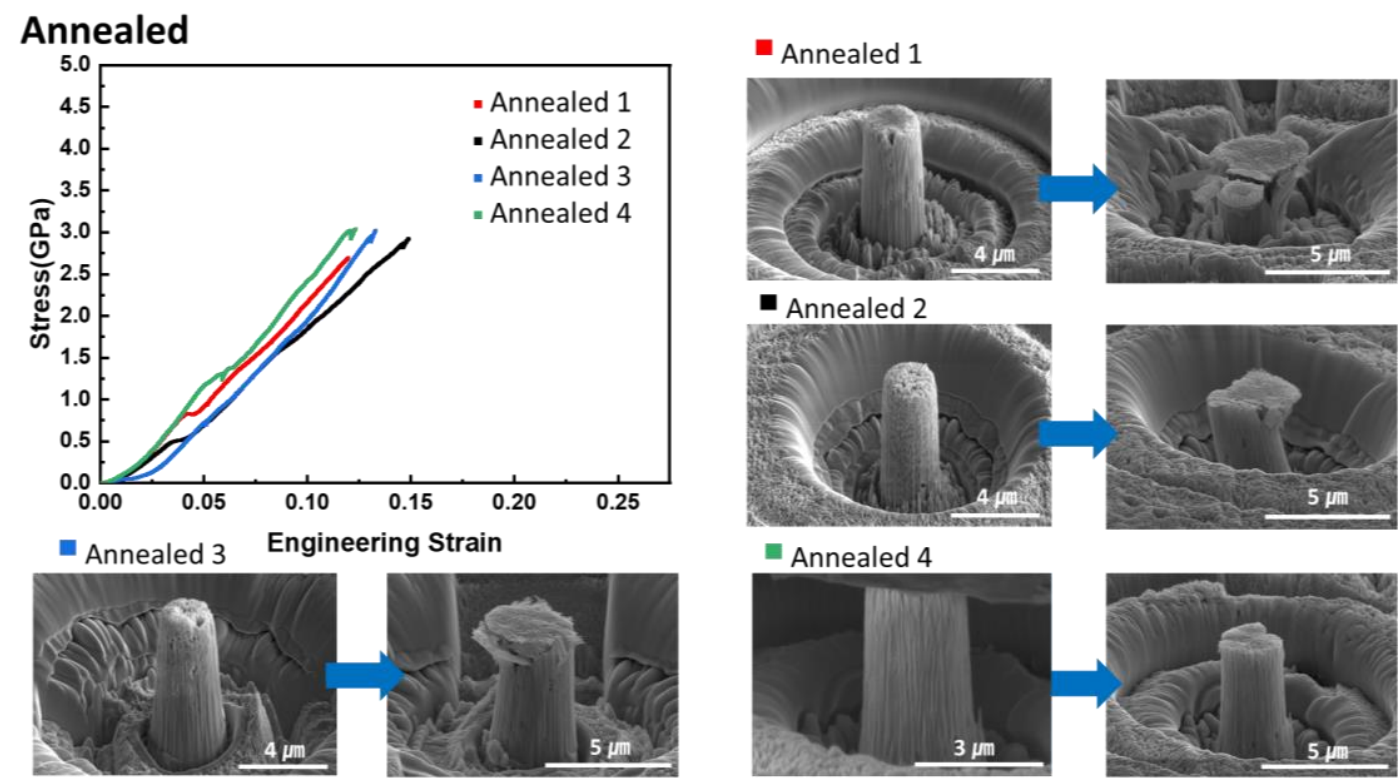

Figure S5. Engineering stress-strain curves of micro-compression test and SEM imag es of before and after compressed micro-pillar for annealed condition. 

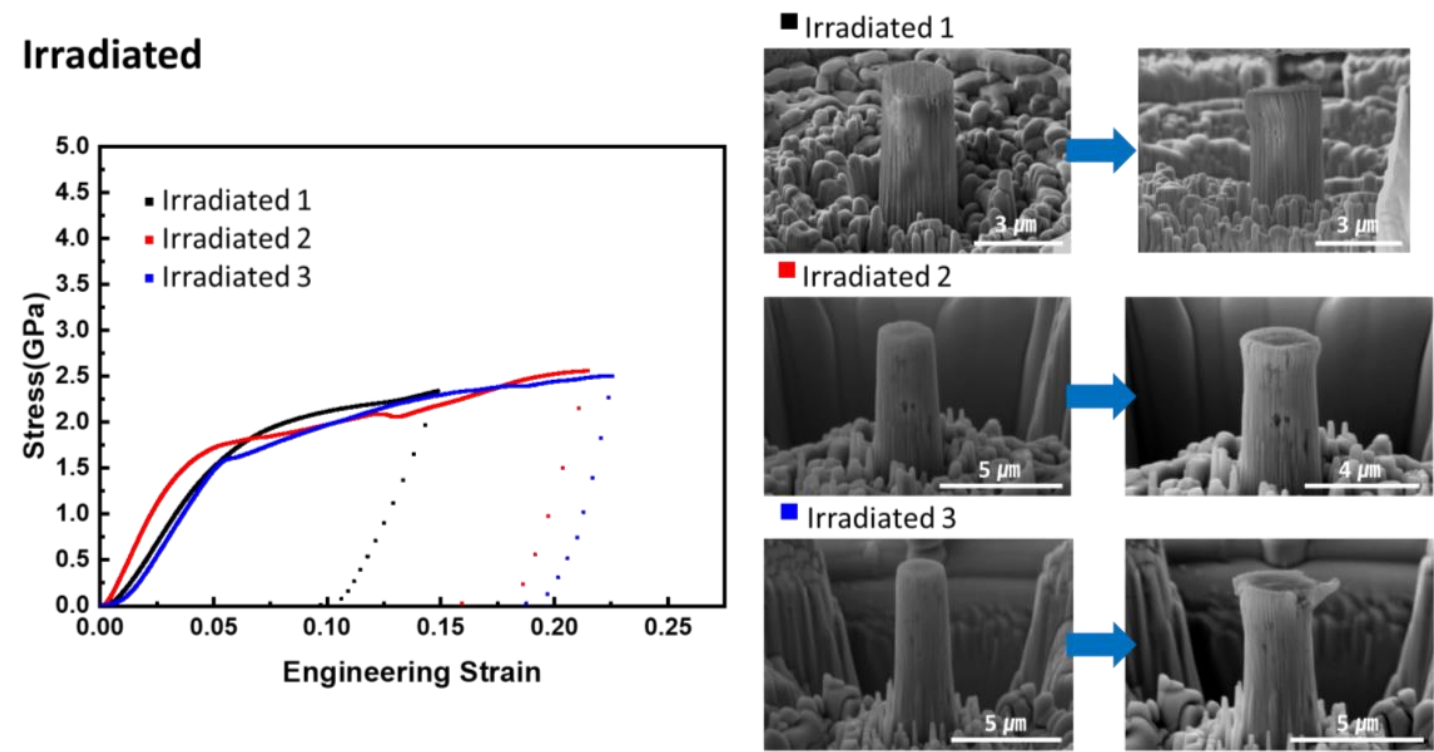

Figure S6. Engineering stress-strain curves of micro-compression test and SEM imag es of before and after compressed micro-pillar for irradiated condition.

\section{Solid}

(a)

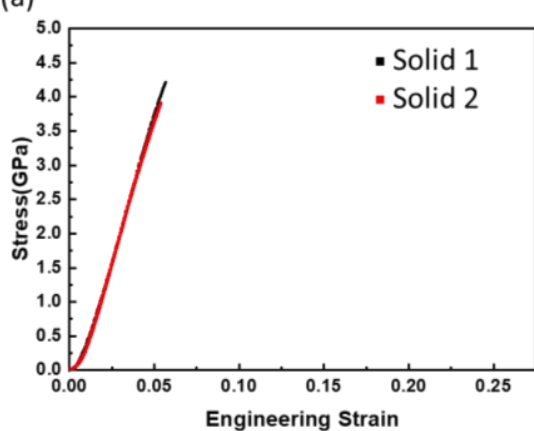

(b)

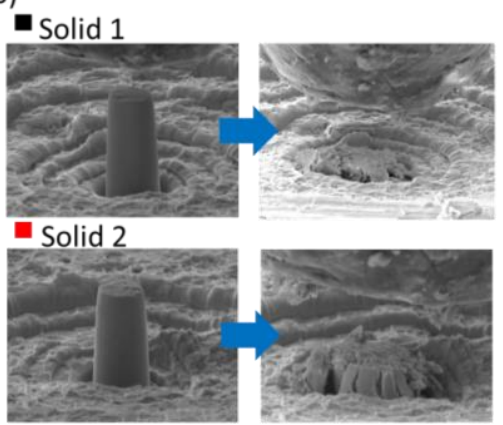

(c)

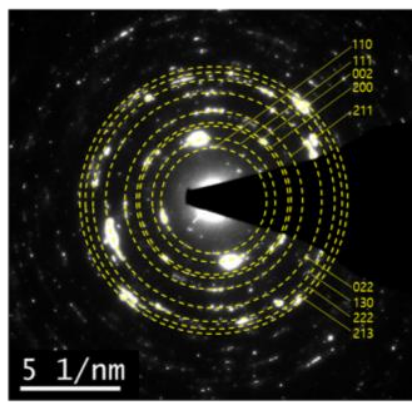

Figure S7. Figure S7. Engineering stress-strain curves of micro-compression test of solid pill ars (a), SEM images of before and after compressed micro-pillar (b) and diffraction pattern of solid zirconia (c).

The results of compression tests under each condition and SEM images of before and after micro-pillars were displayed in Figure S4 through S7. The solid zirconia pillars, which was fabricated without nanoporous structure, exhibited catastrophic failure in compression test (Figure S7b). The crystalline phase of this solid zirconia was indexed with monoclinic zirconia (Figure S7c). 